\title{
Calcineurin Inhibitor-Free Immunosuppression in Renal Transplantation
}

\author{
B. Parada, A. Mota, P. Nunes, F. Macário, J. Pratas, C. Bastos, and A. Figueiredo
}

\begin{abstract}
Purpose. To describe our initial results using a calcineurin inhibitor-free immunosuppression protocol in renal transplants.

Patients and methods. Between October 2001 and June 2003, 56 recipients of a renal allografts were started on an immunosuppression protocol without calcineurin inhibitors, consisting of basiliximab, sirolimus, mycophenolate mofetil, and steroids. We analyzed patient and graft survival, acute rejection episodes, and renal function.

Results. The mean follow-up was 19.6 months. Actuarial patient survival at 1 and 2 years was $98.1 \%$ and $95.3 \%$, respectively. Actuarial graft survival at 1 and 2 years was $92.9 \%$ and $87.6 \%$, respectively. Acute rejection occurred in $27.8 \%$ of the patients, usually Banff 1 $(73.3 \%)$. There was stable renal function with mean serum creatinine of $1.3,1.4,1.3$, and $1.3 \mathrm{mg} / \mathrm{dL}$ at $1,6,12$, and 24 months after transplant.

Conclusions. The use of immunosuppression free of calcineurin inhibitors is effective and safe. Further follow-up is needed to evaluate the impact on long-term results.
\end{abstract}

$\mathrm{K}^{1}$ IDNEY TRANSPLANT OUTCOMES have steadily improved over the past decade $^{1}$ due to modern immunosuppression protocols and better prophylaxis and treatment of common posttransplant infections. ${ }^{2}$ The introduction of calcineurin inhibitors (CNIs)-cyclosporine (CsA) and tacrolimus (Tac) - in the last two decades has resulted in a significant decrease in acute rejection and an improvement in short-term graft survival ${ }^{3}$ with most centers now achieving $85 \%$ to $90 \%$ 1-year graft survival. ${ }^{4}$ However, long-term graft loss due to chronic allograft nephropathy (CAN) remains a major problem in renal transplantation. ${ }^{5}$ While there appear to be many contributors to CAN, many studies emphasize the nephrotoxicity associated with CNIs. ${ }^{6-8}$ Several studies of CNI-free immunosuppression ${ }^{2,9-13}$ or early CsA withdrawal ${ }^{14-16}$ have been reported, with good patient and graft survival and without drug-induced nephrotoxicity. In this report, we describe our initial results using a CNI-free immunosuppression protocol in renal transplants.

\section{PATIENTS AND METHODS}

Between October 2001 and June 2003, 56 recipients of a first renal allograft were enrolled in this prospective study, which had obtained local ethics committee approval and written informed consent from each patient. Exclusion criteria consisted of prior transplantation or exposure to immunosuppressants, type I diabe- ties, severe hyperlipidemia prior to transplantation (serum cholesterol level greater than $350 \mathrm{mg} / \mathrm{dL}$ or serum triglycerides over 400 $\mathrm{mg} / \mathrm{dL}$ ), white blood cells less than $3000 / \mathrm{mm}^{3}$, or platelets less than $100,000 / \mathrm{mm}^{3}$.

Immunosuppressive protocol included basiliximab $20 \mathrm{mg}$ (days 0 and 4), mycophenolate mofetil ( $1 \mathrm{~g}$ two times per day), steroids, and sirolimus (5 mg daily after a $20 \mathrm{mg}$ loading dose). Sirolimus doses were then adjusted to achieve target through levels between 10 and $15 \mathrm{ng} / \mathrm{mL}$ for 6 months and 5 to $10 \mathrm{ng} / \mathrm{mL}$ thereafter. All clinical rejections were biopsy-proven and Banff scored prior to initiation of therapy with high-dose steroids. Primary endpoints of the study were patient survival, graft survival, and the number of acute rejection episodes. Secondary endpoints included delayed graft function, serum creatinine, and calculated creatinine clearance.

Statistical analysis was performed using SPSS for Windows 10.0. Descriptive statistics are presented as mean values and standard deviations for the continuous variables and as frequencies and percentages for the categorical variables. Groups were compared using Student $t$ test for continuous variables and $\chi^{2}$ testing for categorical parameters. Graft and patient cumulative actuarial survivals were calculated by Kaplan-Meier analysis and tested for

From the Department of Urology and Renal Transplantation, University Hospital of Coimbra, Coimbra, Portugal.

Address reprint requests to Belmiro Parada, Department of Urology and Renal Transplantation, University Hospital of Coimbra, 3000 Coimbra, Portugal. E-mail: parada.belmiro@netc.pt

0041-1345/05/\$-see front matter doi:10.1016/j.transproceed.2005.05.044 
Table 1. Patient Characteristics at the Time of Renal Transplantation

\begin{tabular}{lc}
\hline Mean age \pm SD (y) & $39.4 \pm 12.7$ \\
Sex (male/female) & $40 / 6$ \\
Cause of renal failure (\%) & \\
Glomerulonephritis & 31.5 \\
Tubulointerstitial disease & 16.7 \\
Diabetes/hypertension & 14.8 \\
Polycystic disease & 13.0 \\
Other/not determined & 24.0 \\
Graft: cadaveric/living donor & $47(83.9 \%) / 9(16.1 \%)$ \\
HLA mismatches & $3.12 \pm 0.84$ \\
Cold ischemia (h) & $18.1 \pm 7.7$ \\
Donor age $(y)$ & $39.9 \pm 16.2$ \\
\hline
\end{tabular}

differences with the Mantel-Cox log-rank test. Results were considered statistically significant if the $P$ value was less than or equal to .05 .

\section{RESULTS}

Patient characteristics at baseline are shown in Table 1. Mean follow-up was 19.6 months (range: 1 to 32.2 months). Actuarial patient survival at 1 and 2 years was $98.1 \%$ and $95.3 \%$, respectively. Actuarial graft survival at 1 and 2 years was $92.9 \%$ and $87.6 \%$, respectively. There were seven graft losses, two due to chronic dysfunction, two due to vascular complications, and three to death with a functioning graft. When observations were censored for patients dying with a functioning graft, actuarial graft survival at 1 and 2 years was $94.6 \%$ and $91.9 \%$, respectively. Acute rejection occurred in $27.8 \%$ of patients, mostly Banff 1 (73.3\%). Overall, Banff 2 and 3 acute rejection occurred in $7.4 \%$ of the patients. All episodes were reversed with steroids. Actuarial graft survival at 1 and 2 years was $93.3 \%$ and 93.3\%, when acute rejection occurred; and $97.4 \%$ and $89.5 \%$ among patients without acute rejection, a difference that was not statistically significant. There was delayed graft function in $16.7 \%$ of the transplants, but it did not influence graft survival at 1 and 2 years. There was a stable renal function with mean serum creatinine of $1.3,1.4,1.3$, and 1.3 $\mathrm{mg} / \mathrm{dL}$ at $1,6,12$, and 24 months after the transplant. One-year mean serum creatinine level was significantly lower in rejection-free patients $(1.2 \pm 0.4 \mathrm{mg} / \mathrm{dL})$ than in those who suffered an acute rejection $(1.6 \pm 0.8 \mathrm{mg} / \mathrm{dL}, P=$ $.031)$.

\section{DISCUSSION}

Graft survival in renal transplantation is influenced by multiple risk factors, including donor and recipient age, acute rejection, human leukocyte antigen (HLA) mismatches, and comorbidity. ${ }^{15}$ Despite the significant improvements in short-term graft survival and the reduction of acute rejection episodes with CsA and Tac, these CNIs contribute to $\mathrm{CAN} .^{5}$ They decrease glomerular filtration rate, increase blood pressure and hyperlipidemia, and may lead to interstitial fibrosis, with a major negative impact on long-term results. The increasing use of expanded or mar- ginal donors, probably more susceptible to CNI nephrotoxicity, ${ }^{10}$ has emphasized the need to find safer immunosuppressive protocols that both provide protection against acute rejection episodes and permit recovery of graft function after the transplant. ${ }^{2}$ The introduction of sirolimus has allowed the use of immunosuppression protocols without $\mathrm{CNIs}^{2,9-13}$ or with early CsA withdrawal ${ }^{14-16}$ with good patient and graft survival and improved renal histology and function. ${ }^{14}$ In our experience, CNIs free immunosuppression was associated with good 1- and 2-year patient and graft survivals. Although our acute rejection rate $(27.8 \%)$ was slightly higher than some recent reports in the literature, ${ }^{5,15,16}$ the incidence of Banff 2 or 3 acute rejections was low $(7.4 \%)$ and did not influence graft survival. Renal function, as evidenced by serum creatinine values, was stable during our study, although slightly better among rejection-free patients $(1.2 \pm 0.4 \mathrm{mg} / \mathrm{dL})$ than those who suffered acute rejection $(1.6 \pm 0.8 \mathrm{mg} / \mathrm{dL}, P=.031)$.

In conclusion, in our short experience, the use of immunosuppression free of CNIs is effective, with good graft and patient outcomes, and safe. Further follow-up is needed to see the impact of this protocol and of the acute rejection episodes on long-term results.

\section{REFERENCES}

1. Harihan S, Johnson CP, Bresnahan BA, et al: Improved graft survival after renal transplantation in the United States, 1988 to 1996. N Engl J Med 342:605, 2000

2. Flechner SM, Goldfarb D, Moldin C, et al: Kidney transplantation without calcineurin inhibitor drugs: a prospective, randomized trial of sirolimus versus cyclosporine. Transplantation 74:1070, 2002

3. Suwelack B, Gerhardt U, Kobelt U, et al: Design and preliminary results of a randomized study on the conversion of treatment with calcineurin inhibitors to mycophenolate mofetil in chronic renal graft failure: effect on serum cholesterol levels. Transplant Proc 34:1803, 2002

4. Bennett WM, DeMattos A, Meyer MM, et al: Chronic cyclosporine nephropathy: the Achilles' heel of immunosuppressive therapy. Kidney Int 50:1089, 1996

5. Stegall MD, Larson TS, Prieto M, et al: Kidney transplantation without calcineurin inhibitors using sirolimus. Transplant Proc 35(suppl 3A):125S, 2003

6. Bia MJ: Nonimmunologic causes of late renal graft loss. Kidney Int 47:1470, 1995

7. Carpenter CB: Long-term failure of renal transplants: adding insult to injury. Kidney Int 50(suppl):40, 1995

8. Marcen R, Pascual J, Teruel JL, et al: Outcome of cadaveric renal transplant patients treated for 10 years with cyclosporine: is chronic allograft nephropathy the major cause of late graft loss? Transplantation 72:57, 2001

9. Grimbert P, Baron C, Fruchaud G, et al: Long-term results of a prospective randomized study comparing two immunosuppressive regimens, one with and one without CsA, in low-risk renal transplant recipients. Transpl Int 15:550, 2002

10. Shaffer D, Langone A, Nylander WA: A pilot protocol of a calcineurin-inhibitor free regimen for kidney transplant recipients of marginal donor kidneys or with delayed graft function. Clin Transplant 17(suppl 9):31, 2003

11. Hong JC, Kahan BD: A calcineurin antagonist-free induction strategy for immunosuppression in cadaveric kidney transplant recipients at risk for delayed graft function. Transplantation 71: 1320,2001 
12. Groth CG, Bäckman L, Morales JM, et al: Sirolimus (Rapamycin)-based therapy in human renal transplantation. Transplantation 67:1036, 1999

13. Groetzner J, Meiser B, Landwehr P, et al: Mycophenolate mofetil and sirolimus as calcineurin inhibitor-free immunosuppression for late cardiac transplant recipients with chronic renal failure. Transplantation 77:568, 2004

14. Mota A, Arias M, Taskinen EI, et al: Sirolimus-based therapy following early cyclosporine withdrawal provides signifi- cantly improved renal histology and function at 3 years. Am J Transplant 4:953, 2004

15. Kreis H, Oberbauer R, Campistol JM, et al: Long-term benefits with sirolimus-based therapy after early cyclosporine withdrawal. J Am Soc Nephrol 15:809, 2004

16. Oberbauer R, Kreis H, Johnson WG, et al: Long-term improvement in renal function with sirolimus after early cyclosporine withdrawal in renal transplant recipients: 2-year results of the rapamune maintenance regimen study. Transplantation 76:364, 2003 